Jean Telmo Mendoza-Mera; Eugenia Narcisa Arroyo-Carrera; María Valentina Loor-Santos; Mónica Elizabeth Solorzano-Zambrano

http://dx.doi.org/10.35381/r.k.v5i1.869

\title{
Asesoramiento psicopedagógico en el proceso de lectoescritura por estudiantes de educación general básica superior
}

\section{Psychopedagogical counseling in the literacy process by students of higher basic general education}

\author{
Jean Telmo Mendoza-Mera \\ imendoza9668@pucesm.edu.ec \\ Pontificia Universidad Católica del Ecuador, Sede Manabí, Campus Chone \\ https://orcid.org/0000-0002-2698-0057 \\ Eugenia Narcisa Arroyo-Carrera \\ earroyo7577@pucesm.edu.ec \\ Pontificia Universidad Católica del Ecuador, Sede Manabí, Campus Chone \\ https://orcid.org/0000-0003-0773-0981 \\ María Valentina Loor-Santos \\ mloor9413@pucesm.edu.ec \\ Pontificia Universidad Católica del Ecuador, Sede Manabí, Campus Chone \\ https://orcid.org/0000-0001-7724-1951 \\ Mónica Elizabeth Solorzano-Zambrano \\ msolorzano2588@pucesm.edu.ec \\ Pontificia Universidad Católica del Ecuador, Sede Manabí, Campus Chone \\ https://orcid.org/0000-0002-0047-0534
}

Recepción: 05 abril 2020

Revisado: 31 de mayo 2020

Aprobación: 21 junio 2020

Publicación: 01 de julio 2020 
Revista Arbitrada Interdisciplinaria KOINONIA

Año 2020. Vol V. №1. Especial Educación

Hecho el depósito de Ley: FA2016000010

ISSN: 2542-3088

FUNDACIÓN KOINONIA (F.K). Santa Ana de Coro. Venezuela.

Jean Telmo Mendoza-Mera; Eugenia Narcisa Arroyo-Carrera; María Valentina Loor-Santos;

Mónica Elizabeth Solorzano-Zambrano

\title{
RESUMEN
}

La investigación tuvo como objetivo analizar la importancia del asesoramiento psicopedagógico en el proceso de lectoescritura por estudiantes de educación general básica superior del Ecuador, mediante una revisión documental con diseño bibliográfico de trabajos investigativos relacionados a la psicopedagogía y problemas del aprendizaje a través de la lectoescritura, ubicados en revistas arbitradas e indexadas en bases de datos reconocidas por sus buenas practicas editoriales. Se configuran seis indicadores centrales para promover estrategias psicopedagogas en función de promover un efectivo proceso de lectoescritura en estudiantes de educación básica general, lo cual genera una articulación multidisciplinar, por lo tanto, debe abordarse la neurociencia como enfoque promotor de una educación en donde se abordan las redes neuronales y afectivas en razón de estimular la cognición, mediante el uso efectivo de las TIC en razón de promover la investigación y reflexión como eje fundamental del aprendizaje.

Descriptores: Lectura oral; promoción de la lectura; orientación para la lectura; psicología de la educación. (Palabras tomadas del Tesauro UNESCO).

\begin{abstract}
The objective of the research was to analyze the importance of psychopedagogical counseling in the literacy process by students of higher general basic education in Ecuador, through a documentary review with bibliographic design of research works related to psychopedagogy and learning problems through literacy, located in peerreviewed journals and indexed in databases recognized for good editorial practice. Six central indicators are configured to promote psycho-pedagogical strategies in order to promote an effective process of literacy in students of general basic education, which generates a multidisciplinary articulation, therefore, neuroscience must be addressed as a promoter approach to an education where they are addressed neural and affective networks in order to stimulate cognition, through the effective use of ICT in order to promote research and reflection as a fundamental axis of learning.
\end{abstract}

Descriptors: Oral reading; Reading promotion; Reading guidance; Educational psychology. (Words taken from the UNESCO Thesaurus). 
Revista Arbitrada Interdisciplinaria KOINONIA

Año 2020. Vol V. №1. Especial Educación

Hecho el depósito de Ley: FA2016000010

ISSN: 2542-3088

FUNDACIÓN KOINONIA (F.K). Santa Ana de Coro. Venezuela.

Jean Telmo Mendoza-Mera; Eugenia Narcisa Arroyo-Carrera; María Valentina Loor-Santos;

Mónica Elizabeth Solorzano-Zambrano

\section{INTRODUCCIÓN}

La escritura es uno de los procesos de mayor complejidad del ser humano, corresponde a la interrelación con el lenguaje oral, así como de factores neurológicos en compañía del hábito de lectura, así el estudiante se encuentra frente a la posibilidad de escribir correctamente (Cuetos, et al., 2015), en este sentido, tanto docentes como padres, deben acompañar favorablemente al discente en sus inicios escolares con la finalidad de promover el aprendizaje efectivo a través de la lectura y escritura.

Siendo uno de los principales roles formativos el de promover en el estudiante mediante la lectura crítica, la competencia mediática, así podrá fijar posición frente a los diversos eventos sociales e informaciones a las cuales pueda enfrentarse (Briceño-Ruperti, et al., 2019), de ese modo se cumple con la esencia del Currículo de los Niveles de Educación Obligatoria (2016), en donde se indica que:

Se promueve la resolución de problemas por medio del razonamiento lógico y el pensamiento hipotético-deductivo; la participación ciudadana responsable y comprometida con el cuidado de la salud, reproductiva e integral; a través de la comunicación racional y la construcción de acuerdos (p. 44).

El estudiante de Educación Básica General Superior (EBGS) del Ecuador como antesala al bachillerato, debe demostrar competencias y habilidades en lectoescritura en razón del perfil curricular señalado, sin embargo, podrían originarse en un porcentaje que esto no sea exactamente como se plantea, debido a factores relacionados a lo neurológico, afectivo, familiar, interés por el aprendizaje, calidad docente en el acompañamiento escolar, cambios de la edad, búsqueda de ser independientes, entre otros, los cuales podrían incidir negativamente en desarrollar tales capacidades si no existe un adecuado asesoramiento para afrontar asertivamente el proceso de aprendizaje en funcionalidad de la lectoescritura.

Por otro lado, el (Currículo de los Niveles de Educación Obligatoria, 2016), procura en el estudiante la valoración del lenguaje y cultura del Ecuador desde una dimensión intercultural, procesando el empoderamiento de la diversidad social, así se proyecta desde el área de lengua y literatura, la reflexión del entorno como un proceso de 
Jean Telmo Mendoza-Mera; Eugenia Narcisa Arroyo-Carrera; María Valentina Loor-Santos; Mónica Elizabeth Solorzano-Zambrano

crecimiento personal - colectivo en razón de construir el aprendizaje desde una interacción de respeto con sus pares académicos, profundizándose la percepción ética ciudadana, siendo necesaria implementar lecturas críticas que promuevan esta competencia en el estudiante.

De ese modo, es necesario tener en consideración la intersubjetividad para la construcción de conocimientos (Barallobres, 2016), contribuyendo a un proceso lógico de razonamiento en la medida que la interacción con la lectoescritura y compañeros de clase, se podrá promover un adecuado pensamiento mediático, siendo pertinente considerar si los estudiantes sufren impedimentos físicos que impidan tal proceso (Roama-Alves \& Cássia-Nakano, 2015), entre los cuales puede mencionarse la dislexia y dislalia.

La dislexia como problemática que ocasiona inconvenientes para el desarrollo de la lectoescritura de modo efectivo, debe ser abordada a temprana edad con la intención de evitar problemas en el estudiante (Bizama, et al., 2013), tanto de índole personal como académico, para lo cual es conveniente desde una perspectiva multidisciplinar donde se integre la psicopedagogía, neurología, piscología y orientación, tanto para el estudiantes, padres, docentes, esto contribuirá a formalizar un abordaje sinérgico en pertinencia de promover la superación de los problemas que impiden desarrollar la lectoescritura efectivamente (Acta-Caraballo, 2019), siendo recurrente tener en consideración que la dislexia impide la escritura de modo secundario (Escotto-Córdova, 2014).

En el caso del Ecuador, Vélez-Calvo et al. (2015) plantean que en una población de estudiantes de 8 a 13 años "se encontró que el 36\% presentó dislexia superficial, el 33\% dislexia fonológica y el $31 \%$ dislexia mixta", siendo no especificadas las causas que originan las problemáticas planteadas, sin embargo, los autores plantean como alternativa para solucionar la problemática descrita, generar planes de formación en abordaje psicopedagógico como política educativa con la finalidad de formar a los docentes en estrategias con fines a minimizar el impacto negativo.

Por otro lado (Scrich-Vázquez, et al., 2017), enfatizan que la baja estima, problemas de motivación, interés por el aprendizaje, son problemas comunes en estudiantes 
Jean Telmo Mendoza-Mera; Eugenia Narcisa Arroyo-Carrera; María Valentina Loor-Santos; Mónica Elizabeth Solorzano-Zambrano

ecuatorianos con problemas de dislexia, disgrafía y discalculia, siendo además perjudicial que son literalmente tratados como incapaces, desfavoreciendo aún más, el acercamiento del estudiante hacia el estudio, proyectándose problemas en concretar un aprendizaje significativo.

En complemento a lo planteado, la investigación tuvo como finalidad realizar una revisión documental con diseño bibliográfico de trabajos investigativos relacionados a la psicopedagogía y problemas del aprendizaje a través de la lectoescritura, ubicados en revistas arbitradas e indexadas en bases de datos reconocidas por sus buenas practicas editoriales, por lo que se gestionó como objetivo analizar la importancia del asesoramiento psicopedagógico en el proceso de lectoescritura por estudiantes de educación general básica superior del Ecuador.

\section{Asesoramiento psicopedagógico en el proceso de lectoescritura por estudiantes de educación general básica superior}

La dificultad de lectoescritura si es de índole biológico, acompañará al estudiante a lo largo de su vida, siendo minimizado por medio de terapias clínicas y psicopedagógicas (Ríos-Flórez \& López-Gutiérrez, 2017), por otro lado, (Jiménez-Jiménez \& Marques, 2018), consideran que el sistema cognitivo al verse afectado, afecta las labores escolares del estudiante, siendo posible aplicar programas de intervención clínica que contribuyen a superar los inconvenientes, siendo pertinente evaluar el grado de deterioro cognitivo con la finalidad de diseñar el abordaje pertinente a aplicar.

En relación a lo planteado, (Bravo, 2018) indica que "los conocimientos derivados de las neurociencias de la educación y fundamentadas en neuroimágenes muestran una estrecha relación entre los procesos cognitivos cerebrales y la psicopedagogía del aprendizaje escolar" (p. 1), por lo que el aprender a leer correctamente, corresponde a dos escenarios, el biológico y el psicopedagógico, se necesita un óptimo funcionamiento neuropsicológico así como de la concepción social de aprendizaje en la medida que se promueve un efectivo aprendizaje, siendo necesario por parte del docente combinar 
Revista Arbitrada Interdisciplinaria KOINONIA

Año 2020. Vol V. №1. Especial Educación

Hecho el depósito de Ley: FA2016000010

ISSN: 2542-3088

FUNDACIÓN KOINONIA (F.K). Santa Ana de Coro. Venezuela.

Jean Telmo Mendoza-Mera; Eugenia Narcisa Arroyo-Carrera; María Valentina Loor-Santos;

Mónica Elizabeth Solorzano-Zambrano

estimulación cerebral con estrategias psicopedagógicas en razón a las características de la audiencia.

La formación sobre el abordaje de las problemáticas neurológicas que afectan el aprendizaje, son necesarias realizarlas, siendo de gran interés el de involucrar a los padres y familia, por cuanto estos constituyen el estímulo y refuerzo con la finalidad de cooperar con el docente en trabajar coordinadamente en tal fin (Webster, et al., 2019), el sistema educativo debe intensificar la efectividad de las escuelas de padres o familia como un aliado en la consecución de aplicar programas de intervención para superar las dificultades escolares (Hernández, 2018).

El proceso de escritura es complejo, siendo necesario promover habilidades resuntivas con la finalidad de que el estudiante precise la construcción de escritos con coherencia desde el pensamiento lógico, implicando que un texto académico por lo general contiene introducción, desarrollo y cierre, siendo necesaria promover competencias para lograr en efectividad tal objetivo (Dovale, 2018), entendiéndose lo resuntivo con la oportunidad de crear a través de la escritura, para lo cual debe existir conjugación entre la educación y la neuroeducación para tal fin.

En este sentido (Burgos \& Osses, 2015), exhortan a incluir los avances neurocientíficos en relación a la cognición, los cuales deben ser conocidos por quienes se forman en la carrera educativa, así como los docentes en ejercicio a través de la formación continua, de ese modo, existirá mayor probabilidad de promover una educación de calidad e inclusiva, abordando al estudiante asertivamente en función de incentivar sus habilidades, fortalezas, constituyendo la ruta hacia un aprendizaje significativo, considerándose para tal fin, una escuela basada en la formación integral (Kosunen \& Hansen, 2018).

En ese sentido, es necesario actualizar a los docentes, padres, sobre el abordaje psicopedagógico de los estudiantes en función de generar una lectoescritura creativa en concordancia con transcender los factores que inciden negativamente en la generación del aprendizaje, siendo pertinente abordar una educación fundamentada desde la investigación acción como medio para la integración y transformación de los actores 
Jean Telmo Mendoza-Mera; Eugenia Narcisa Arroyo-Carrera; María Valentina Loor-Santos; Mónica Elizabeth Solorzano-Zambrano

educativos (Nieto, 2016), lo cual contribuye a superar la enseñanza del modelo tradicional al de la pedagogía discursiva de la escritura (Borregales, 2020).

Comprendiéndose como el paso de trascender la enseñanza de decodificación a la de creación reflexiva mediante la lectoescritura en conjugación al nivel cognitivo y académico del estudiante (Chacha-Supe \& Rosero-Morales, 2020), propiciándose una educación basada en la investigación y reflexión para lograr tal fin (Smeyers, 2019), siendo pertinente involucrar la lectoescritura a través de las redes sociales, viéndose los estudiantes motivados a leer y escribir en virtud de identificarse con la tecnología (Rosenberg, et al., 2016).

Siendo indispensable promover en los estudiantes, la orientación necesaria para evitar hábitos tóxicos, por cuanto estos se intensifican cuando se tienen problemas de aprendizaje especifico (Fernández-Díaz, et al., 2017), lo cual advierte la urgencia de corregir a temprana edad los inconvenientes de aprendizaje, así el sistema educativo contribuye a la formación de un ciudadano reflexivo, transcendiendo el modelo basado netamente en la repetición mecánica del conocimiento, en este sentido, (Molano, 2018) advierte que la superación de los problemas específicos de aprendizaje pueden ser superados por medio del enfoque afectivo cognitivo.

Es imprescindible por medio de la formación continua que el docente se forme en métodos de lectura emergente, propiciando las capacidades y competencias para aprender por medio de la reflexión, de la indagación, de la creación analítica, proceso que se perfecciona a lo largo de la vida (Conejo \& Carmiol, 2017), siendo lo más complicado aprender a aplicar efectivamente estrategias relacionadas a lo emocional, así como la integración de la familia al acompañamiento pedagógico (Dabdub-Moreira \& Pineda-Cordero, 2015), en complemento se debe tener en consideración que lo emocional no solo contribuye a mejorar el aprendizaje en el estudiante, sino, a su socialización (Rubiales, et al., 2018).

Las TIC es un elemento que debe ser tenido en cuenta para configurar estrategias de asesoramiento psicopedagógico en función de promover desde esta área, la inclusión de los estudiantes con diversidades especiales, esto por medio de la adaptación tecnológico 
Jean Telmo Mendoza-Mera; Eugenia Narcisa Arroyo-Carrera; María Valentina Loor-Santos; Mónica Elizabeth Solorzano-Zambrano

y software en favor de lo académico (Zambrano-Pincay, et al., 2019), por otro lado, (Arroyo-Carrera, et al., 2020), complementan al indicar que por medio de las nuevas herramientas tecnológicas se incentiva el aprendiza creativo, siendo indispensable aplicarla con fines de lectoescritura en coordinación de superar los problemas específicos de aprendizaje.

Así los juegos educativos desarrollados en plataformas tecnológicas, contribuyen al aprendizaje asertivo, promoviendo la motivación en el estudiante, aunado que pueden ser programados a las necesidades particulares de cada discente, por consiguiente, se proyecta la posibilidad de promover el asesoramiento psicopedagógico en función de las ventajas que ofrece la tecnología de la información en la sociedad del conocimiento.

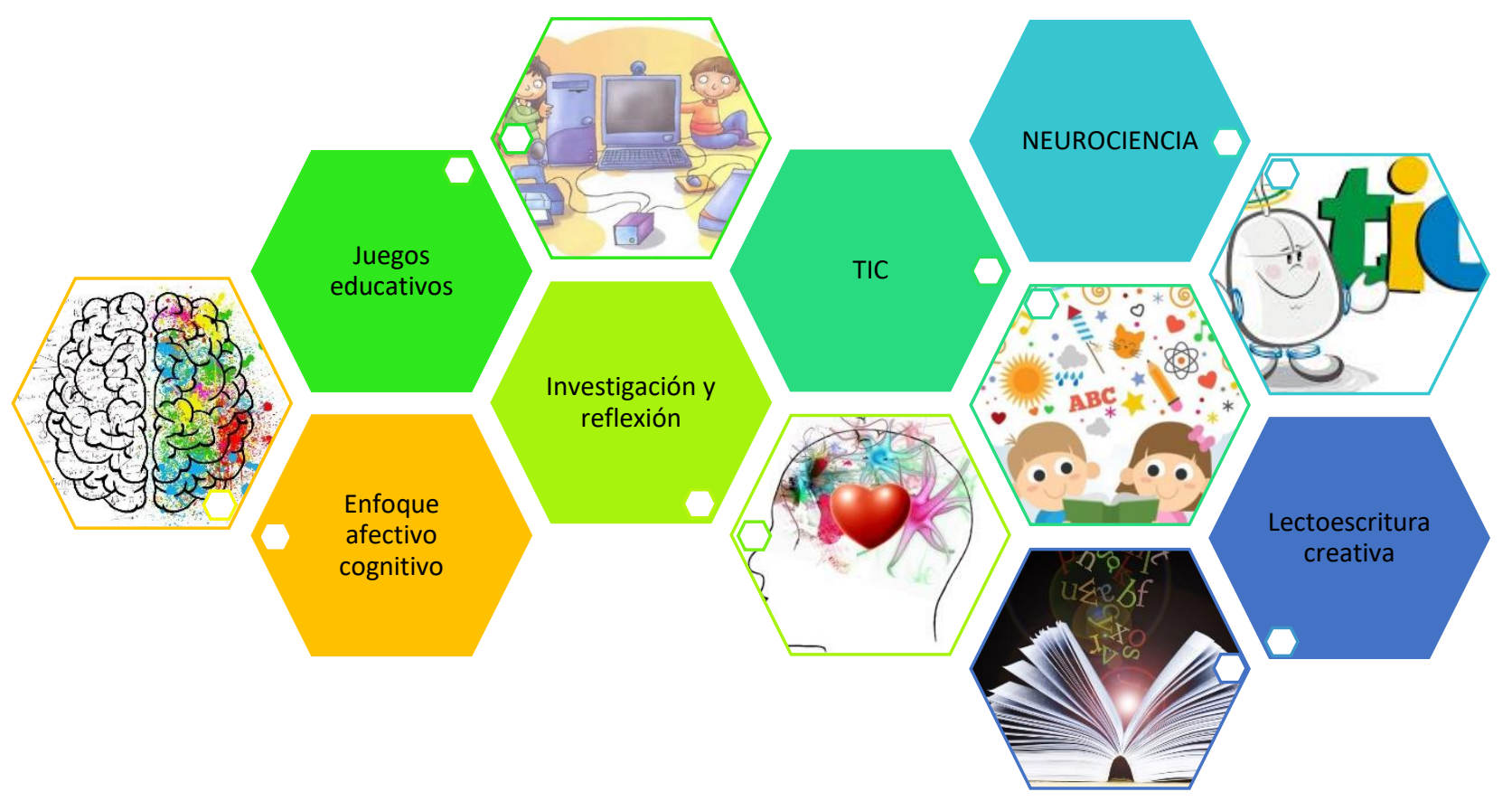

Figura 1. Indicadores de lectoescritura desde la psicopedagogía. 
Jean Telmo Mendoza-Mera; Eugenia Narcisa Arroyo-Carrera; María Valentina Loor-Santos; Mónica Elizabeth Solorzano-Zambrano

En función de la literatura revisada, se configuran seis indicadores centrales para promover estrategias psicopedagogas en función de promover un efectivo proceso de lectoescritura en estudiantes de educación básica general, lo cual genera una articulación multidisciplinar, por lo tanto, debe abordarse la neurociencia como enfoque promotor de una educación en donde se abordan las redes neuronales y afectivas en razón de estimular la cognición, mediante el uso efectivo de las TIC en razón de promover la investigación y reflexión como eje fundamental del aprendizaje, apoyándose en herramientas lúdicas para promover la lectura - escritura en funcionalidad comprensiva, entendiendo que los problemas biológicos que puedan afectar a los discentes, deben abordarse complementariamente con la finalidad de ser incluidos, así el sistema educativo incentiva una educación de calidad e inclusiva en equidad de condiciones.

\section{CONCLUSIÓN}

El asesoramiento psicopedagógico debe generarse desde la integración multidisciplinar con la finalidad de abordar integralmente al estudiante, tanto a quienes padecen problemas biológicos en detrimento de la lectura y lenguaje, así como quienes no lo tienen, por cuanto se requiere educar en sintonía en promover la afectividad como un proceso que contribuya al estudiante a conocerse así mismo, a tomar confianza, a valorarse mediante una estima asertiva, esto requiere del apoyo del equipo docente, padres - representantes, e incluso de contar con políticas educativas afines a tal fin, por cuanto el estudiante debe ser concebido como la generación de relevo, la cual debe asumir los retos globales en la mayor pertinencia posible, para lo cual, la escuela como espacio socializador, contribuye a la conformación de ciudadanos óptimos al servicio de la humanidad.

\section{FINANCIAMIENTO}

No monetario. 
Revista Arbitrada Interdisciplinaria KOINONIA

Año 2020. Vol V. №1. Especial Educación

Hecho el depósito de Ley: FA2016000010

ISSN: 2542-3088

FUNDACIÓN KOINONIA (F.K). Santa Ana de Coro. Venezuela.

Jean Telmo Mendoza-Mera; Eugenia Narcisa Arroyo-Carrera; María Valentina Loor-Santos;

Mónica Elizabeth Solorzano-Zambrano

\section{AGRADECIMIENTO}

A la Pontificia Universidad Católica del Ecuador, Sede Manabí, Campus Chone, por motivar el desarrollo de esta investigación

\section{REFERENCIAS}

Acta-Caraballo, Y. (2019). Modelo de formación neuroeducativa para docentes en la República Dominicana. [An Educational Neuroscience-Based Teaching Model for Dominican Teachers]. Revista Cubana de Educación Superior, 38(3).

Arroyo-Carrera, E., Loor-Santos, M., Mendoza-Mera, J., \& Solorzano-Zambrano, M. (2020). Gestión de aprendizaje creativo mediante la Herramienta Powtoon en estudiantes de lengua y literatura. [Creative learning management through the Powtoon Tool in language and literature students]. EPISTEME KOINONIA, 3(5), 253-269. http://dx.doi.org/10.35381/e.k.v3i5.775

Barallobres, G. (2016). Diferentes interpretaciones de las dificultades de aprendizaje en matemática. [Different interpretations of mathematics learning difficulties]. Educación matemática, 28(1), 39-68.

Bizama M, Marcela, Arancibia, B, \& Sáez, K. (2013). Intervención Psicopedagógica Temprana en Conciencia Fonológica como Proceso Metalingüístico a la base de la Lectura en niños de 5 a 6 años socialmente vulnerables. [Early psychopedagogical intervention in phonological awareness as a metalinguistic process based on Reading, in socially challenged children between 5-6 years old]. Estudios pedagógicos (Valdivia), 39(2), 25-39. https://dx.doi.org/10.4067/S0718$\underline{07052013000200002}$

Borregales, L. (2020). Génesis y evolución socio-histórica de la macro categoría cultura escrita. [Genesis and socio-historical evolution of the macro category written $\begin{array}{llll}\text { culture]. } & \text { EPISTEME } & \text { KOINONIA, } & 3(5),\end{array}$ http://dx.doi.org/10.35381/e.k.v3i5.529

Bravo, L. (2018). El Paradigma de las Neurociencias de la Educación y el Aprendizaje del Lenguaje Escrito: Una Experiencia de 60 Años. [The Paradigm of Educational Neuroscience and the Learning of the Written Language: Sixty Years After]. Psykhe (Santiago), 27(1), 1-11. https://dx.doi.org/10.7764/psykhe.27.1.1101 
Jean Telmo Mendoza-Mera; Eugenia Narcisa Arroyo-Carrera; María Valentina Loor-Santos; Mónica Elizabeth Solorzano-Zambrano

Briceño-Ruperti, M., Vallejo-Valdivieso, P., \& Moya-Martínez, M. (2019). Estructuras mentales y competencia mediática en el aprendizaje significativo. [Mental structures and media competence in meaningful learning]. CIENCIAMATRIA, 5(9), 680-695. https://doi.org/10.35381/cm.v5i9.258

Burgos, C, \& Osses, S. (2015). Creatividad y neurociencia: binomio esencial en el contexto educativo. [Creativity and Neuroscience: An essential binomial in educational context]. Revista médica de Chile, 143(7), 948949. https://dx.doi.org/10.4067/S0034-98872015000700018

Chacha-Supe, M., \& Rosero-Morales, E. (2020). Procesos iniciales de enseñanza aprendizaje de la lectoescritura en los estudiantes del nivel de preparatoria. [Initial teaching-learning processes of literacy in high school students]. Revista Arbitrada Interdisciplinaria Koinonía, 5(9), 311-336. http://dx.doi.org/10.35381/r.k.v5i9.650

Conejo, L., \& Carmiol, A. (2017). Conocimientos sobre la lectoescritura emergente y prácticas en las aulas para su promoción: Un estudio con docentes de Educación preescolar en Costa Rica. [Emergent Literacy Knowledge and Classroom Practices for its promotion: a Study with Preschool Education Teachers in Costa Rica]. Revista Costarricense de Psicología, 36(2), 105-121. http://dx.doi.org/10.22544/rcps.v36i02.02

Cuetos, F., Suárez-Coalla, P., Molina, M. I., \& Llenderrozas, M. C. (2015). Test para la detección temprana de las dificultades en el aprendizaje de la lectura y escritura. [Test for the early detection of difficulties in learning to read and write]. Pediatría Atención Primaria, 17(66), e99-e107. https://dx.doi.org/10.4321/S1139$\underline{76322015000300002}$

Currículo de los Niveles de Educación Obligatoria (2016). Educación General Básica. Sub Nivel Superior. [Basic general education. Upper Level Sub]. Recuperado de https://bit.ly/32QPymm

Dabdub-Moreira, M., \& Pineda-Cordero, A. (2015). La atención de las necesidades educativas especiales y la labor docente en la escuela primaria. [Response to Special Educational Needs and Teaching Efforts in Elementary School]. Revista Costarricense de Psicología, 34(1), 41-55.

Dovale, Y. (2018). Las habilidades resuntivas de ensayos académicos expositivos en estudiantes de la Universidad Nacional Experimental Francisco de Miranda. [The summary skills of expository academic essays in students of the Francisco de Miranda National Experimental University]. EPISTEME KOINONIA, 1(1), 18-34. http://dx.doi.org/10.35381/e.k.v111.488 
Revista Arbitrada Interdisciplinaria KOINONIA

Año 2020. Vol V. №1. Especial Educación

Hecho el depósito de Ley: FA2016000010

ISSN: 2542-3088

FUNDACIÓN KOINONIA (F.K). Santa Ana de Coro. Venezuela.

Jean Telmo Mendoza-Mera; Eugenia Narcisa Arroyo-Carrera; María Valentina Loor-Santos; Mónica Elizabeth Solorzano-Zambrano

Escotto-Córdova, E. (2014). Intervención de la lectoescritura en una niña con dislexia. [Literacy intervention in a girl with dyslexia]. Pensamiento Psicológico, 12(1),55-69.

Fernández-Díaz, Y., Ortiz-Martínez, M., Aguilar-Valdés, J., Pérez-Sosa, D., \& Serra-Larín, S. (2016). Hábitos tóxicos en adolescentes y jóvenes con problemas de aprendizaje, 2011-2013. Revista Cubana de Salud Pública, 43(1).

Hernández, A. (2018). Transitando por el camino de la escuela para padres, madres y representantes. Una experiencia vivida. [Walking on the way to school for parents and representatives. A lived experience]. EPISTEME KOINONIA, 1(1), 51-71. http://dx.doi.org/10.35381/e.k.v1i1.490

Jiménez-Jiménez, S, \& Marques, D. (2018). Impacto de la intervención neuropsicológica infantil en el desarrollo del sistema ejecutivo. Análisis de un caso. [Impact of Child Neuropsychological Intervention on the Development of the Executive System. Case Study]. Avances en Psicología Latinoamericana, 36(1), 11-28.

Kosunen, S., \& Hansen, P. (2018). Discursive narratives of comprehensive education politics in Finland. [Narrativas discursivas de la política educativa integral en Finlandia]. European Educational Research Journal, 17(5), 714-732. https://doi.org/10.1177/1474904118764938

Molano, G. (2018). Dificultades de aprendizaje y su incidencia en la adolescencia. [Learning difficulties and their incidence in adolescence]. Revista Prisma Social, (23), 366-387.

Nieto, M. (2016). Learner corpora, corpora of professional translations and creative writing in a course on translation of general texts: an action research project. [Corpus de aprendices, corpus de traducciones profesionales y escritura creativa en la clase de traducción general: un proyecto de investigación-acción]. Cadernos de Tradução, 36(1), 121-146. https://doi.org/10.5007/2175-7968.2016v36nesp1p121

Ríos-Flórez, J., \& López-Gutiérrez, C. (2017). Neurobiología de los trastornos del aprendizaje y sus implicaciones en el desarrollo infantil: propuesta de una nueva perspectiva conceptual. [Neurobiology of learning disorders and their implications for child development: proposal of a new conceptual perspective]. Revista Psicoespacios, (11)19, 1-20.

Roama-Alves, R, \& Cássia-Nakano, T. (2015). Criatividade em indivíduos com transtornos e dificuldades de aprendizagem: revisão de pesquisas. [creativity in individuals with learning disabilities and disorders: research review]. Psicologia Escolar e Educacional, 19(1), 87-96. https://doi.org/10.1590/2175$\underline{3539 / 2015 / 0191802}$ 
Jean Telmo Mendoza-Mera; Eugenia Narcisa Arroyo-Carrera; María Valentina Loor-Santos; Mónica Elizabeth Solorzano-Zambrano

Rosenberg, J. M., Greenhalgh, S. P., Koehler, M. J., Hamilton, E. R., \& Akcaoglu, M. (2016). An investigation of State Educational Twitter Hashtags (SETHs) as affinity spaces. [Una investigación de los hashtags educativos estatales de Twitter (SETH) como espacios de afinidad]. E-Learning and Digital Media, 13(1-2), 24-44. https://doi.org/10.1177/2042753016672351

Rubiales, J., Russo, D., Paneiva Pompa, J., \& González, R. (2018). Revisión sistemática sobre los programas de Entrenamiento Socioemocional para niños y adolescentes de 6 a 18 años publicados entre 2011 y 2015/Systematic Review of SocialEmotional Training Programs For Children and Adolescents. Revista Costarricense de Psicología, 37(2), 163-186. http://dx.doi.org/10.22544/rcps.v37i02.05

Scrich-Vázquez, A., Ruz-Fonseca, L., Bembibre-Mozo, D., \& Torres-Céspedes, I. (2017). La dislexia, la disgrafia y la discalculia: sus consecuencias en la educación ecuatoriana. [Dyslexia, dysgraphia and dyscalculia and psychological disorders of learning: teaching attitude in Ecuador]. Archivo Médico Camagüey, 21(1), 6-12.

Smeyers, P. (2019). How to characterize research and scholarship that matters for the educational field? [¿Cómo caracterizar la investigación y la investigación que son importantes para el campo educativo?]. European Educational Research Journal, 18(5), 622-635. https://doi.org/10.1177/1474904119865857

Vélez-Calvo, X., Tárraga-Mínguez, R., Fernández-Andrés, M., Sanz-Cervera, P., Blázquez-Garcés, J., \& Tijeras-lborra, A. (2015). Incidencia de la dislexia en ecuador: relación con el ci, lateralidad, sexo y tipo de escuela. [Incidence of dyslexia in Ecuador: relationship with IC, laterality, sex and type of school]. Revista INFAD de Psicología. International Journal of Developmental and Educational Psychology., 2(1), 249-258. https://doi.org/10.17060/ijodaep.2015.n1.v2.64

Webster, F, Piedra, M, \& Estévez, F. (2019). Percepción de los padres de niños con déficit ejecutivos que presentan dificultades en el aprendizaje de matemáticas. [Parental perception in children with executive deficits who have learning difficulties in mathemathics]. Revista Ecuatoriana de Neurología, 28(3), 52-58.

Zambrano-Pincay, G., Vallejo-Valdivieso, P., Vallejo-Pilligua, P., \& Bravo-Cedeño, G. (2019). Los profesionales de la Psicopedagogía en la atención a la diversidad como Agente Educativo. [Professionals of Psychopedagogy in attention to diversity as an Educational Agent]. Revista Arbitrada Interdisciplinaria de Ciencias de la Salud. Salud y Vida, 3(6), 41-57. http://dx.doi.org/10.35381/s.v.v3i6.304 
Revista Arbitrada Interdisciplinaria KOINONIA

Año 2020. Vol V. №1. Especial Educación

Hecho el depósito de Ley: FA2016000010

ISSN: 2542-3088

FUNDACIÓN KOINONIA (F.K). Santa Ana de Coro. Venezuela.

Jean Telmo Mendoza-Mera; Eugenia Narcisa Arroyo-Carrera; María Valentina Loor-Santos; Mónica Elizabeth Solorzano-Zambrano

2020 por los autores. Este artículo es de acceso abierto y distribuido según los términos y condiciones de la licencia Creative Commons Atribución-NoComercial-Compartirlgual 4.0 Internacional (CC BY-NC-SA 4.0)

(https://creativecommons.org/licenses/by-nc-sa/4.0/). 